\title{
Artificial Multisensory Neuron with Fused Haptic and Temperature Perception for Multimodal In-Sensor Computing
}

\author{
Qingxi Duan ${ }^{1}$, Teng Zhang ${ }^{1}$, Chang Liu ${ }^{1}$, Rui Yuan ${ }^{1}, \mathrm{Ge} \mathrm{Li}^{2}$, Pek Jun Tiw ${ }^{1}$, Ke Yang ${ }^{1}$, \\ Chen $\mathrm{Ge}^{2}$, Ru Huang ${ }^{1}$, and Yuchao Yang ${ }^{1}$ \\ ${ }^{1}$ Peking University \\ ${ }^{2}$ Chinese Academy of Sciences
}

March 7, 2022

\begin{abstract}
Human receives and transmits various information from the outside world through different sensory systems. The sensory neurons integrate various sensory inputs into a synthetical perception to monitor complex environments, and this fundamentally determines the way how we perceive the world. Developing multifunctional artificial sensory elements that can integrate multisensory perception plays a vital role in future intelligent perception systems, whereas prior spiking neurons reported can only handle single-mode physical signals. Here, we present a bio-inspired haptic-temperature fusion spiking neuron based upon a serial connection of piezoresistive sensor and $\mathrm{VO}_{2}$ volatile memristor. The artificial sensory neuron is capable of detecting and encoding pressure and temperature inputs based on the voltage dividing effect and the intrinsic thermal sensitivity of metal-insulator transition in $\mathrm{VO}_{2}$. Recognition of Braille characters is achieved through multiple piezoresistive sensors, taking advantage of the spatial integration capabilities of such spiking neurons. Notably, the traditionally separate haptic and temperature signals can be fused physically in the sensory neuron when synchronizing the two sensory cues, which is able to recognize multimodal haptic/temperature patterns. The artificial multisensory neuron thus provides a promising approach towards e-skin, neuro-robotics and human-machine interaction technologies.
\end{abstract}

Corresponding author(s) Email: ruhuang@pku.edu.cn yuchaoyang@pku.edu.cn

\section{ToC Figure}

Here a bio-inspired haptic-temperature fusion spiking neuron based on a combination of piezoresistive sensor and $\mathrm{VO}_{2}$ volatile memristor is demonstrated. The thermal characteristics of $\mathrm{VO}_{2}$ memristor and mechanical sensory properties of the piezoresistive sensor are exploited and combined in the same component, enabling multimodal in-sensor computing. This could provide a promising approach towards e-skin, neurorobotics and human-machine interaction technologies. 

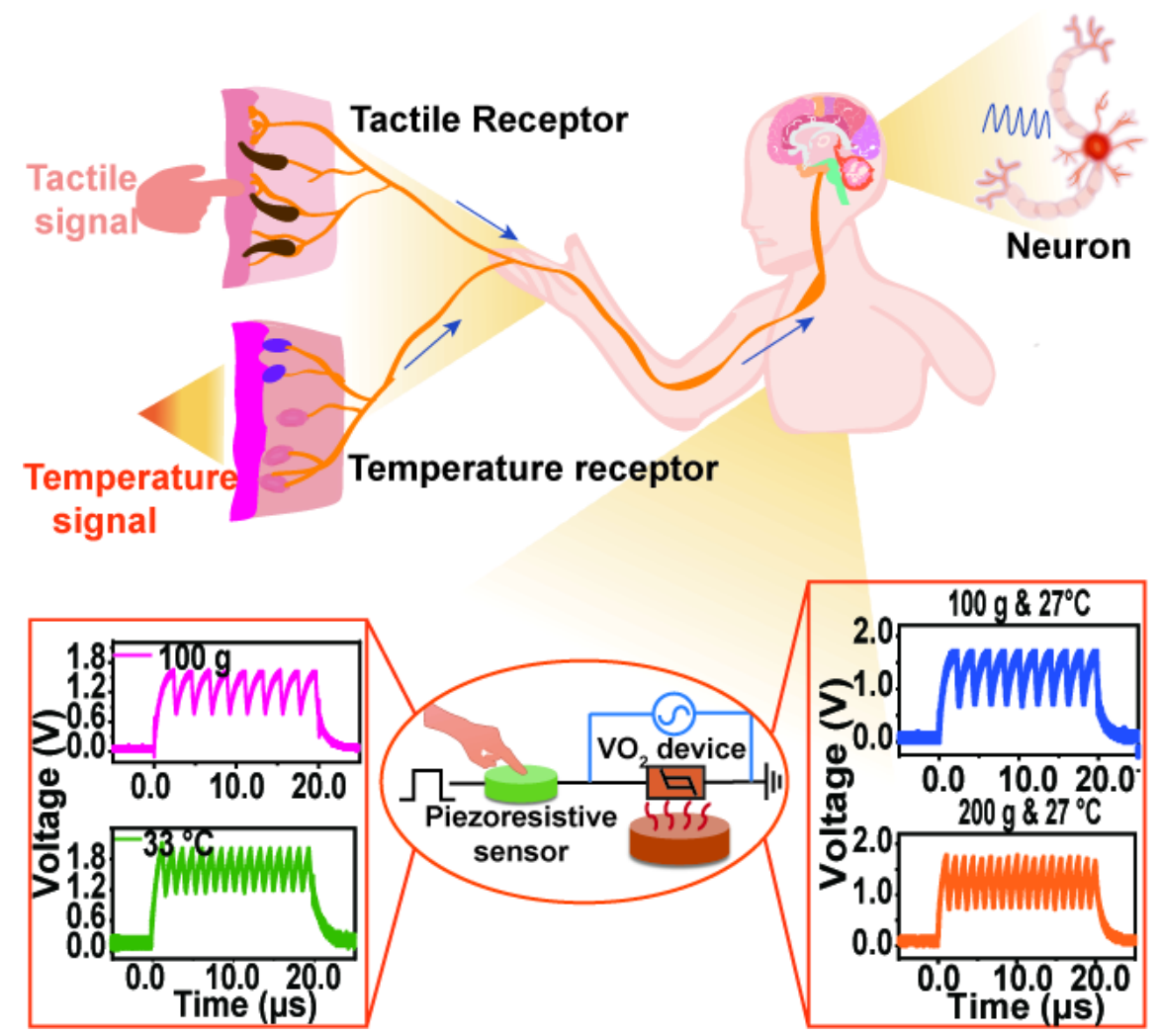

\section{Introduction}

The sensory system is a part of the nervous system that processes sensory information, which includes receptors, neural pathways, and the sensory center of the cerebral cortex. In the sensory neural network of humankind, the sensory receptors convert environmental information into potential changes and encode such potential changes into spike trains with neural spike coding in the cell body. Subsequently, interneurons convey the spike trains from the receptors to the cerebral cortex of the brain, where the information is decoded into sensory perceptions for further processing. ${ }^{[1-6]}$ Such structure forms the basis of sensing, pre-processing and encoding capabilities of the human sensory system. This outperforms digital computers in dealing with a large number of complex tasks, such as perception and real-time sensory data processing. Therefore, it is of great significance to draw inspiration from the human sensory system and develop artificial sensory hardware that can efficiently realize the perception and encoding capabilities, which will provide a promising approach towards e-skin, neurorobotics and human-machine interaction technologies.

Traditional complementary metal oxide semiconductor (CMOS) technology utilizes complex auxiliary circuits and bulky capacitors to emulate sensors and bio-dynamics, which takes up a large area and high computational cost. ${ }^{[6-10]}$ In recent years, many researchers have devoted efforts to emulating the synaptic dynamics or neuronal behaviors using emerging devices such as non-volatile memristors, ${ }^{[11-13]}$ volatile memristors ${ }^{[14,15]}$ and synaptic transistors. ${ }^{[16,17]}$ Meanwhile, there were recent reports on emulation of biological sensing functions by integrating functional sensors with synaptic and neuron components. ${ }^{[18-28]}$ One way is to use sensors combined with artificial synapse devices, ${ }^{[19-27]}$ and recently, volatile memristors have emerged as excellent candidates for the construction of artificial sensory neurons due to their simple two-terminal structure and dynamic threshold switching (TS) characteristics. [18, 23, 28, 29, 32] However, aiming at sensory neuron, these prior studies only focus on single-mode sensory perception, but rarely achieves the integration of multiple sensory inputs, ${ }^{[28-32]}$ which is distant from the efficient processing of cross-sensory information 
in biology. Therefore, it is of great significance to construct an artificial sensory system that not only senses and converts the physical information in real time, but also directly fuse and integrate multisensory inputs in a hardware-efficient manner.

Here, we report an artificial multisensory neuron consisting of a piezoresistive sensor and a $\mathrm{VO}_{2}$ based volatile memristor connected in series. Such artificial sensory neurons can be used to sense different pressure inputs and convert them into spike trains as a result of the voltage dividing effect between the piezoresistive sensor and $\mathrm{VO}_{2}$ memristor. Besides, Tthe spiking neuron is also capable of sensing temperature, by taking advantage of the intrinsic thermal sensitivity of metal-insulator transition in $\mathrm{VO}_{2}$. The spiking neuron is utilized to recognize Braille characters using multiple piezoresistive sensors. Notably, the traditionally separate haptic and temperature signals can now be fused physically in the $\mathrm{VO}_{2}$ based sensory neuron when synchronizing the two sensory cues, which is able to recognize multimodal haptic/temperature patterns. Such multisensory neurons could provide a promising approach towards e-skin, neuro-robotics and human-machine interaction technologies.

\section{Experimental Section/Methods}

Fabrication of $\mathrm{VO}_{2}$ volatile memristor: The $20 \mathrm{~nm} \mathrm{VO}_{2}$ films were epitaxially grown on c- $\mathrm{Al}_{2} \mathrm{O}_{3}$ substrates by pulsed-laser deposition (PLD) technique using a 308-nm XeCl excimer laser operated at an energy density of about $1 \mathrm{~J} / \mathrm{cm}^{2}$ and a repetition rate of $3 \mathrm{~Hz}$. The $\mathrm{VO}_{2}$ films were deposited at $530{ }^{\circ} \mathrm{C}$ in a flowing oxygen atmosphere at the oxygen pressure of $2.0 \mathrm{~Pa}$. Then, the films were cooled down to the room temperature at the speed of $20{ }^{\circ} \mathrm{C} / \mathrm{min}$. The deposition rate of $\mathrm{VO}_{2}$ thin films was calibrated by X-ray Reflection (XRR). All $\mathrm{VO}_{2}$ devices studied in this work were fabricated on $\mathrm{Al}_{2} \mathrm{O}_{3}$ substrates. First, $\sim 10 \mathrm{~nm}$ single crystal $\mathrm{VO}_{2}$ film serving as the switching layer was deposited by pulsed laser deposition (PLD). Afterwards, $\sim 5 \mathrm{~nm}$ thick Ti was deposited as the electrodes and capped by $\sim 40 \mathrm{~nm}$ thick Au protection layer by e-beam evaporation, where the patterning of the electrodes was done by electron beam lithography and lift-off processes.

Microstructural and compositional characterization: The TEM samples in this work were prepared by the focused ion beam (FIB) technique using a dual-beam FIB system (FEI Helios Nanolab workstation). During FIB patterning, the sample was first coated by $\mathrm{SiO}_{2}$ and $\mathrm{Pt}$ layer deposited using the electron beam to avoid surface damages, followed by higher-rate Pt coating using normal ion beam process that served as majority of the protective layer during FIB cutting. TEM and STEM images as well as EDS measurements were performed on FEI Tecnai F20 and the HRTEM and SAED results were analyzed by the Digital Micrograph software (Gatan Inc.). The SEM characterization was conducted on a field emission SEM (Merlin Compact).

Electrical measurements: All the electrical measurements were performed using an Agilent B1500A semiconductor parameter analyzer and the RIGOL MSO8104 digital storage oscilloscope. Voltage pulses were applied by the Agilent B1500A. We used an Agilent B1500A semiconductor parameter analyzer to perform electrical measurements of a single $\mathrm{VO}_{2}$ device in Fig. 2g-h, 4b, 6b and Figure S2-6, S9, S11, S12. In Figs. 3-6 and Figure S14, Agilent B1500A is applied to create the pulse signal, and one channel of the oscilloscope is used to measure the output of Agilent B1500A, while the other channel measures the voltage of the output node in the neuron circuit.

Simulations: A COMSOL Multiphysics package finite element simulation was used to analyze the electroinduced phase change and thermal distribution of the film. The 3D structure and material properties of the simulation model, including test electrodes, substrates, and $\mathrm{VO}_{2}$ composite films, which were the same as those of the experiment. In this simulation, the fitted electrical characteristics of the device is set according to the real test data (see Figure 2f).

The Multi-layer-perceptron with an architecture of $2 \times 20 \times 11$ and $3 \times 20 \times 11$ were simulated in MATLAB, that is, 2 or 3 input neurons for dataset inputs, 20 hidden neurons and 11 output neurons for possible classes. Each output neuron represents one of the combinations of pressure and temperature. The neural network was trained online with Backpropagation (BP) algorithm. 
Piezoresistive sensor: The piezoresistive sensors used in this study is RP-C18.3-LT. The detailed technical and physical properties are as follows:

\begin{tabular}{ll}
\hline Thickness & $0.4 \mathrm{~mm}$ \\
Shape & Flexible \\
Actuation force & $20 \mathrm{~g}$, Res. $<=200 \mathrm{k} \Omega$ \\
Operation range & $20 \mathrm{~g}$ to $6000 \mathrm{~g}$ \\
Resolution & continuous \\
Non-actuated resistance & $>10 \mathrm{M} \Omega$ \\
Response time & $<10 \mathrm{~ms}$ \\
Life time & $>1 \mathrm{million}$ \\
Repeatability same part & $+/-3 \%$, Average R@1000 g \\
Repeatability part to part & $+/-10 \%$, Average R@1000 g \\
Hysteresis & $+10 \%$, 1000 g \\
EDS & Not ESD sensitive \\
\hline
\end{tabular}

\section{Results}

\section{Oscillation neuron based on $\mathrm{VO}_{2}$ volatile memristor}

The perception and cognition ability of human brain assisted with associative biomechanical and temperature sensations are critical for acquiring somatosensory information. The brain encloses numerous neurons to receive the interactive signals in different modalities (e.g., mechanical, temperature signals) and implements cross-modal neuromorphic computation in the multisensory association area. ${ }^{[3,34]}$ Figure 1 presents the biological multisensory integration nervous system, and the corresponding artificial multisensory system that is constructed (as shown in the orange dashed box), which consists of a piezoresistive sensor and $\mathrm{VO}_{2}$-based oscillation neuron.

As one of the key components, the oscillation neuron was first built, and its characteristics were thoroughly analyzed. As schematically illustrated in Figure 2a, the oscillation neuron consists of two $\mathrm{Au} / \mathrm{Ti}$ electrodes sandwiching a $\mathrm{VO}_{2}$ film in a lateral device structure. The structure of the device is characterized by the scanning electron microscopy (SEM) image in Figure $2 \mathrm{~b}$, showing that the channel length of the device is approximately $400 \mathrm{~nm}$. Figure $2 \mathrm{c}$ and $2 \mathrm{~d}$ show transmission electron microscopy (TEM) images of the $\mathrm{VO}_{2}$ device, with spatial mapping of $\mathrm{Al}, \mathrm{Au}, \mathrm{Ti}, \mathrm{V}$ and $\mathrm{O}$ elements using energy-dispersive X-ray spectroscopy (EDS). An EDS line scan of the cell is shown in Figure S1, Supporting Information. Figure 2e exhibits a high-resolution TEM image of the $\mathrm{VO}_{2}$ layer. The clear lattice fringes and the corresponding Fast Fourier Transformation (FFT) result (Figure 2f) show that the $\mathrm{VO}_{2}$ has high-quality crystalline structure with a tetragonal phase.

Electrical measurements of the $\mathrm{Au} / \mathrm{Ti} / \mathrm{VO}_{2} / \mathrm{Ti} / \mathrm{Au}$ memristors show that the devices have threshold switching (TS) characteristics without going through any electroforming process. As shown in Figure $2 \mathrm{~g}$, reliable TS characteristics can be obtained under voltage sweeping mode with 100 cycles. Specifically, when the applied voltage exceeds a threshold voltage $\left(V_{\text {th }}\right)$ of $\sim 1.4 \mathrm{~V}$, the $\mathrm{VO}_{2}$ device switches from high resistance state (HRS) to low resistance state (LRS), and automatically returns to HRS once the applied voltage drops below a holding voltage ( $V_{\text {hold }}$ ) of $\sim 1.0 \mathrm{~V}$ (Figure $2 \mathrm{~g}$, Figure S3 further shows the stable TS characteristics without compliance current). Transient electrical measurements show that the switching speed of the $\mathrm{VO}_{2}$ device is $<120 \mathrm{~ns}$ from off state to on state, and $<50 \mathrm{~ns}$ from on state to off state (Figure S2, Supporting Information). We also examined the stability and uniformity of the $\mathrm{VO}_{2}$ memristor, including the endurance, the cycle-to-cycle and device-to-device variations of the device, which demonstrate that the $\mathrm{VO}_{2}$ memristors have endurance of $10^{6}$ cycles as well as acceptable cycle-to-cycle and device-to-device variations (Figure 


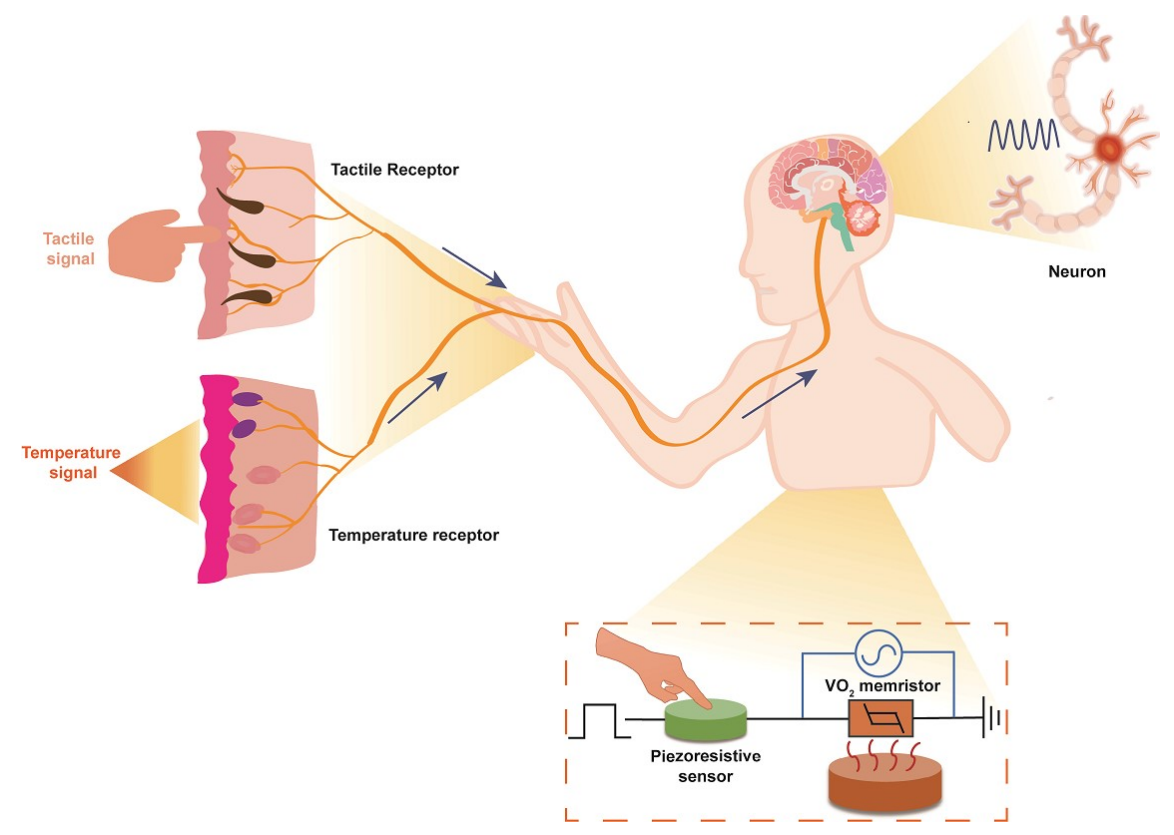

Figure 1: Schematic diagram of the biological and artificial multisensory neurons. A biological multisensory neuron that is stimulated by pressure and temperature. Pressure applied onto mechanoreceptors change the potentials of receptors that are embedded in the skin. Temperature applied onto thermal receptor change the receptor potential. The cell body of the sensory neuron integrates the potentials and initiates spikes with coded pressure information and temperature information. Inside the orange dashed box, it is the schematic images of the artificial multisensory neuron consist of a piezoresistive sensor and $\mathrm{VO}_{2}$-based oscillation neuron.

S4-S6, Supporting Information), making them qualified for functioning as oscillatory neurons.

The TS characteristics in $\mathrm{VO}_{2}$ volatile memristors can be well interpreted by the Mott transition coupled with a structural phase transition. ${ }^{[35-37]}$ Figure $2 \mathrm{~h}$ schematically depicts the dynamic evolution of the device state during the threshold switching process through COMSOL simulation. The orange line shows the experimental current-voltage $(I-V)$ characteristics, while the bule line depicts the simulation curve. From state (1) to state (2), heat is generated in $\mathrm{VO}_{2}$ while the applied voltage increases. When the applied voltage exceeds $V_{\text {th }}$, joule heating generated by the voltage induces formation of a filament through the $\mathrm{VO}_{2}$ gap, leading to a transition from HRS to LRS, and the filament expands from the channel to both sides as the process progresses (state (2) to state (4)). When the applied voltage decreases, the heat is gradually dissipated and the size of the filament gradually decreases, and the device undergoes a transition from state (5) to state (8), leading to switching from LRS to HRS.

Based on the threshold switching characteristics in $\mathrm{VO}_{2}$ memristors, oscillatory neuronal behavior can be implemented with a simple circuit, whose configuration is depicted in Figure 3a. The $\mathrm{VO}_{2}$ volatile memristor is connected in series with a load resistor $\left(R_{\mathrm{L}}\right)$, and the intrinsic capacitance of the $\mathrm{VO}_{2}$ memristor provides the dynamics for integration. Figure 3d shows the oscillation characteristics of the spiking neuron. When a suitable voltage $\left(V_{\text {in }}\right)$ is applied and a matched serial resistor $\left(R_{\mathrm{L}}\right)$ is connected, the voltage dividing effect between the $R_{\mathrm{L}}$ and $\mathrm{VO}_{2}$ memristor results in a voltage drop over $\mathrm{VO}_{2}$ memristor that exceeds its $V_{\text {th }}$. Therefore, the $\mathrm{VO}_{2}$ device will switch from off to on state. Once the device is in on state, the voltage drops across $R_{\mathrm{L}}$ and the $\mathrm{VO}_{2}$ device will be re-distributed, and the voltage gets lower than $V_{\text {hold }}$ and hence the device returns to off state. To achieve such oscillation effect, the following requirements should be satisfied:

$$
\frac{V_{i n} \cdot R_{o f f}}{R_{o f f}+R_{L}} \geq V_{t h}
$$



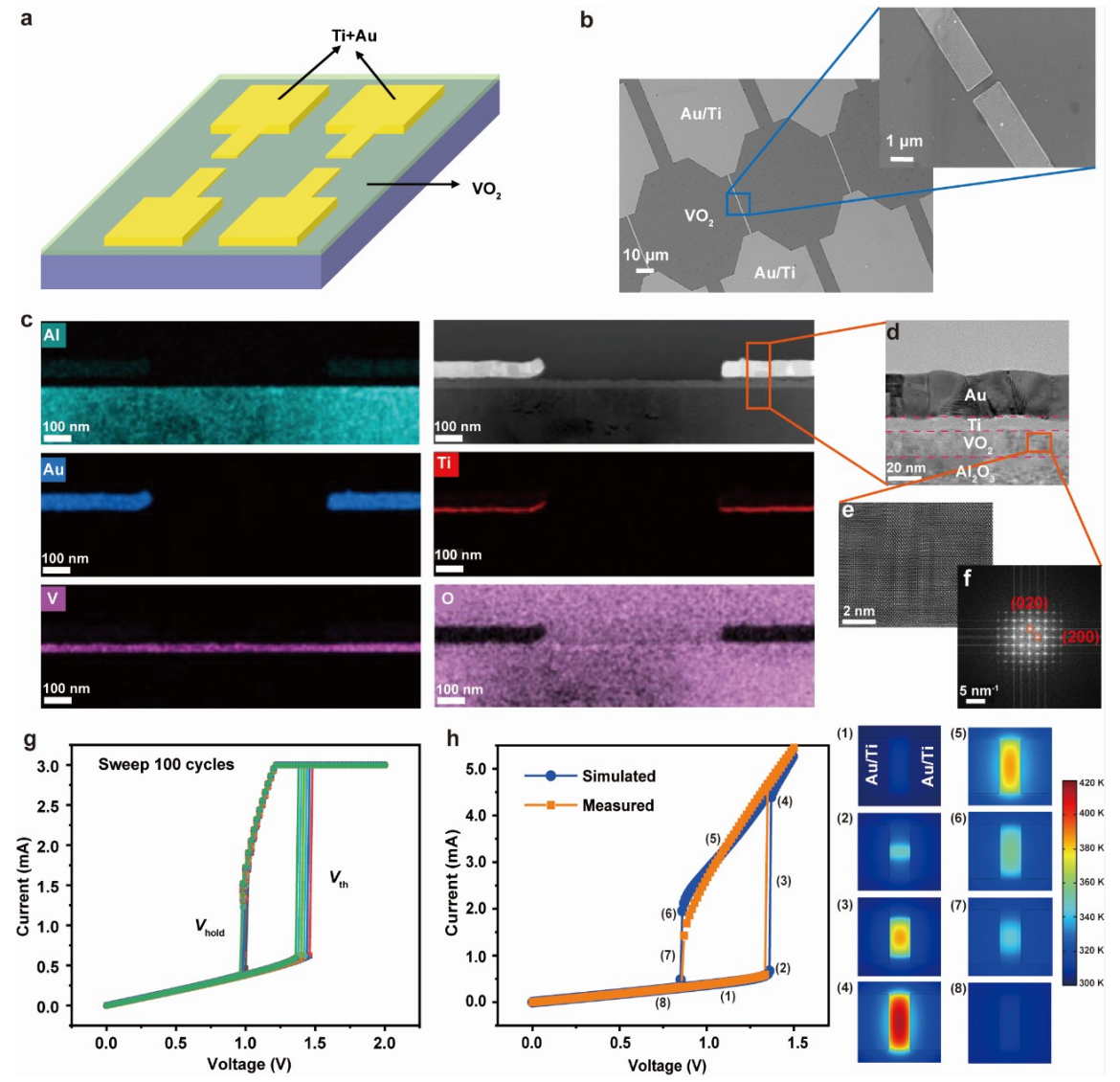

Figure 2: $\mathbf{V O}_{2}$ volatile memristor analysis. a) Schematic diagram of the memristive device, which consists of two $\mathrm{Au} / \mathrm{Ti}$ electrodes sandwiching a dielectric film $\left(\mathrm{VO}_{2}\right)$ in a lateral device structure. b) Scanning electron microscopy (SEM) image of the $\mathrm{VO}_{2}$ device. c) Cross-sectional transmission electron microscopy (TEM) image and the elemental mapping of the materials in the system for $\mathrm{Al}, \mathrm{Au}, \mathrm{Ti}, \mathrm{V}$ and $\mathrm{O}$, respectively. d) Cross-sectional TEM image of the $\mathrm{VO}_{2}$ device. e) High-resolution TEM image of the $\mathrm{VO}_{2}$ layer. f) The diffraction pattern extracted by Fourier transform of Figure 2e. g) Current-voltage $(I-V)$ characteristics of the device repeated for 100 cycles. h) Simulated $I-V$ curves from the thermodynamic simulation. The right area of figure show heat distribution at each moment.

$$
\frac{V_{\text {in }} \cdot R_{o n}}{R_{\text {on }}+R_{L}} \leq V_{\text {hold }}
$$

When Equations (1) and (2) are satisfied, the above spike event will be produced and repeated (Figure 3d). Figure 3d and 3e show the oscillating behavior of the spiking neuron, when the $R_{\mathrm{L}}$ or input voltage is varied, respectively. As expected, the oscillation frequency decreases $(0.9,0.7,0.55,0.35 \mathrm{MHz})$ as $R_{\mathrm{L}}$ increases $(3.0,3.6,4.2,4.8 \mathrm{k} \Omega$ ) (Figure $3 \mathrm{~d}$ ), whereas the rate increases $(0.45,0.65,0.8,0.9 \mathrm{MHz})$ as the input voltage increases $(4.2,4.8,5.4,6.0 \mathrm{~V}$ ) (Figure 3e). The dependence of the oscillation frequency on the load resistor $\left(R_{\mathrm{L}}\right)$ is systematically tested under different input voltages $(4,5,6 \mathrm{~V})$, and the results are summarized in Figure 3b, further demonstrating that the oscillation frequency decreases as $R_{\mathrm{L}}$ increases in each $V_{\text {in }}$ case. Similarly, Figure 3c systematically analyzes the dependence of the oscillation frequency on $V_{\text {in }}$, when varied $R_{\mathrm{L}}(3.0,3.5,4.0,4.5 \mathrm{k} \Omega)$ is adopted, implying the same increasing trend of oscillation frequency with increase of $V_{\text {in }}$. Therefore, the oscillation frequency of the spiking neuron can be effectively modulated by the threshold voltage of the $\mathrm{VO}_{2}$ memristor and circuit parameters $\left(R_{\mathrm{L}}\right.$ and $\left.V_{\text {in }}\right)$, which paves the way for the construction of an artificial sensory system. 

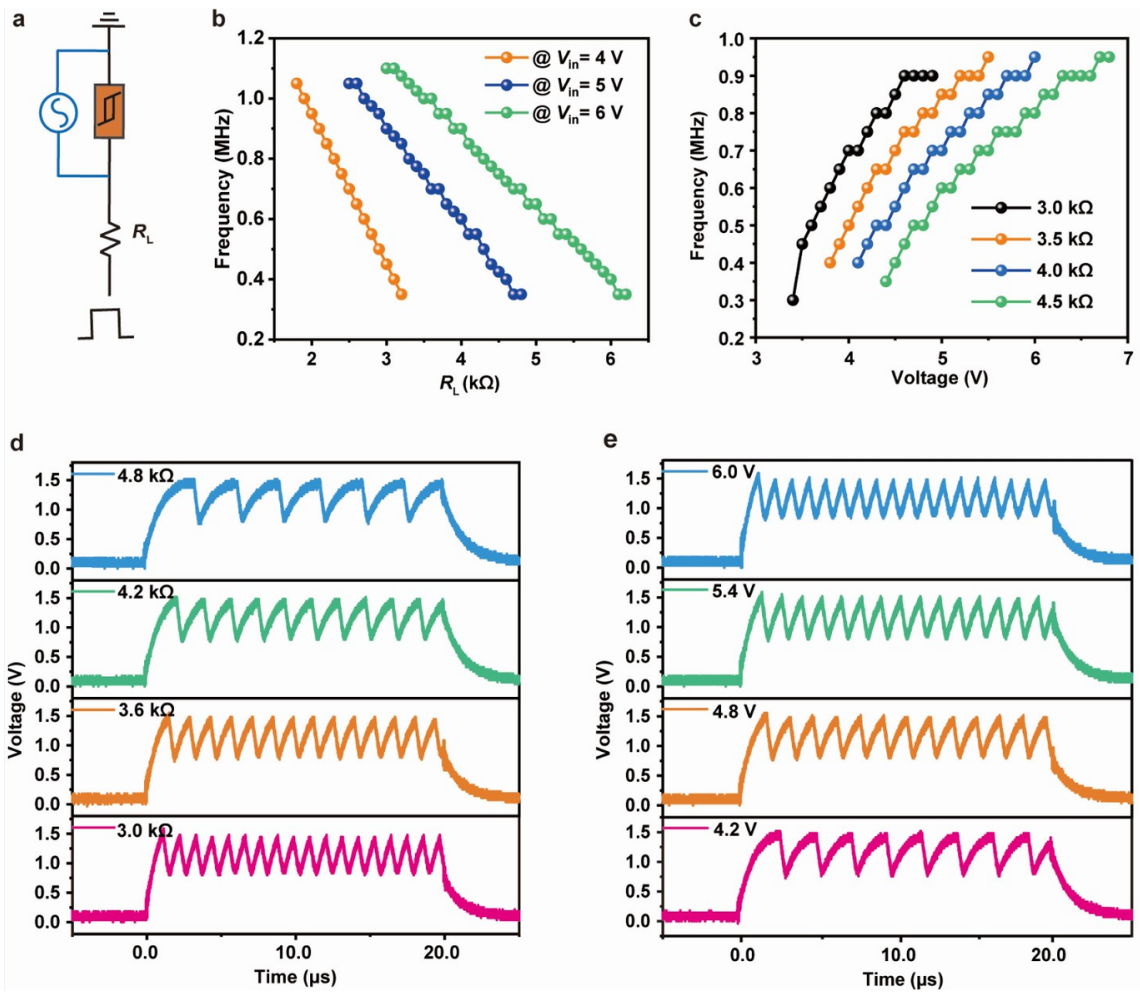

Figure 3: Oscillation characteristics based on $\mathbf{V O}_{2}$ volatile memristor. a) Schematic diagram of the oscillation neuron. b) Oscillation frequency as a function of load resistance $\left(R_{\mathrm{L}}\right)$ with different input voltages $(4,5,6 \mathrm{~V})$. c) Oscillation frequency as a function of input voltage $\left(V_{\text {in }}\right)$ with different load resistors $(3.0,3.5,4.0,4.5 \mathrm{k} \Omega)$. d) Neuronal response of the oscillation neuron under a constant bias voltage $(5 \mathrm{~V}, 20$ $\mu \mathrm{s})$ and the influence of varying resistance $\left(R_{\mathrm{L}}\right)$. The output frequencies are $0.9,0.7,0.55$, and $0.35 \mathrm{MHz}$ at $R_{\mathrm{L}}$ of $3.0,3.6,4.2,4.8 \mathrm{k} \Omega$, respectively. e) Neuronal response of the oscillation neuron in series with a fixed resistance $\left(R_{\mathrm{L}}=4 \mathrm{k} \Omega\right)$ and the influence of varying input voltage $\left(V_{\mathrm{in}}\right)$. The output frequencies are $0.45,0.65,0.8$, and $0.9 \mathrm{MHz}$ at $V_{\text {in }}$ of $4.2,4.8,5.4$, and $6.0 \mathrm{~V}$, respectively.

\section{Artificial haptic perception neuron based on $\mathrm{VO}_{2}$ volatile memristor}

In a biological sensory nervous system, the mechanoreceptors are responsible for sensory information transduction. When the external stimuli exceed the mechanical threshold of the mechanoreceptors, sensory information is being coded through an action potential at a certain frequency. ${ }^{[38,39]}$ To mimic the biological activity from mechanoreceptors, the spiking response due to a haptic event, we integrated the piezoresistive sensor with a $\mathrm{VO}_{2}$ memristor to emulate the haptic perception as an artificia mechanoreceptor. Given the dependence of the output frequency of $\mathrm{VO}_{2}$ oscillation neuron on the load resistor (Figure 2b,d), the haptic perception function can be achieved by replacing the fixed $R_{\mathrm{L}}$ with a piezoresistive sensor. Figure 4a shows a schematic diagram of the artificial haptic perception neuron using $\mathrm{VO}_{2}$ volatile memristor and a commercially available piezoresistive sensor (the entire experimental setup is shown in Figure S7, Supporting information). Figure $4 \mathrm{~b}$ shows the $I-V$ curves of the piezoresistive sensor under different pressures, showing different resistance state in response to pressure/weight inputs (from 20 to $700 \mathrm{~g}$ ). Moreover, Figure 4c summarizes the resistance response of the piezoresistive sensor under different pressures/weights. The stability and thermal characteristics of the piezoresistive sensor are further shown in Figures S8 and S9, Supporting 
Information. It can be clearly seen that the resistance gradually decreases as the pressure increases. This characteristic can be generalized by a power function:

$$
\mathrm{y}=\alpha \mathrm{x}^{-\beta}(3)
$$

where $\alpha=95570, \beta=-0.64$ are extracted by fitting the curve presented in Figure 4c. The range of resistance change under pressure is between $\sim 1 \mathrm{k} \Omega$ and $\sim 20 \mathrm{k} \Omega$, which meets the series resistance required by the $\mathrm{VO}_{2}$ oscillation neuron. This piezoresistive sensor can thus be combined with the $\mathrm{VO}_{2}$ oscillation neuron to emulate artificial haptic perception, where the sensor is used as a receiver of pressure signals and the resultant sensory signal is converted into spike trains by the $\mathrm{VO}_{2}$ neuron.

Indeed, when different pressures/weights $(100,150,200,250 \mathrm{~g})$ are applied to the sensor with a constant bias voltage $(5 \mathrm{~V}, 20 \mu \mathrm{s})$, the oscillation neuron exhibits different output spike frequencies $(0.45,0.55,0.75$, $0.95 \mathrm{MHz}$ ), as shown in Figure $4 \mathrm{~d}$. The converted spike frequency increases as the pressure increases. In this way, the artificial haptic sensory neuron can directly respond to pressure signals and encode them into spikes, and the output spikes can then transmit information to spiking neural networks for further processing.

Haptic perception allows human to recognize objects, discriminate texture, and react appropriately in a social exchange. ${ }^{[2,40]}$ This essentially requires integration of multiple spatial correlated sensory stimuli. In order to achieve this, we combine two sensors in parallel as a proof of concept, and they are further connected in series with a $\mathrm{VO}_{2}$ memristor (the circuit structure is shown in Figure S10, Supporting Information). This is utilized to recognize Braille characters in the present study. As shown in Figure 4e, the black circles represent convex patterns in the Braille characters, while the white circles indicate no convex, which correspond to the cases of sensing pressure and no pressure when being touched, respectively. These scenarios were emulated in experiment by applying $100 \mathrm{~g}$ for convex patterns and $0 \mathrm{~g}$ otherwise. The results in Figure 4e show that when only one of the two sensors are triggered, the output oscillation frequency of $\mathrm{VO}_{2}$ neuron is $\sim 0.4 \mathrm{MHz}$. However, when the two sensors are triggered at the same time, the output oscillation frequency will be higher $(\sim 1.1 \mathrm{MHz})$. The output frequency is zero when neither of the sensors is triggered (detailed information is shown in Supplementary Video 1-3). Therefore, the Braille characters can be read out from the different patterns of output frequencies produced by the $\mathrm{VO}_{2}$ neurons. It should be pointed out that some Braille characters may not be fully distinguished based on the horizontal inputs, and in this case an additional process can be introduced to apply vertical inputs onto the device so as to further distinguish them.

\section{Artificial temperature perception neuron based on $\mathrm{VO}_{2}$ volatile memristor}

In human sensory system, temperature sensation is also an indispensable sensory ability, besides tactile sensation, which can help the human body respond to the temperature of the outside world so that the central nervous system can initiate a motor response, for example, avoiding injury of the human body. The $\mathrm{VO}_{2}$ device is inherently sensitive to temperature due to its thermally-driven metal-insulator transition, ${ }^{[1,42]}$ and hence this feature can be exploited to construct an artificial temperature perception neuron, as illustrated in Figure 5a. Figure 5b firstly shows the $I-V$ characteristics of $\mathrm{VO}_{2}$ volatile memristor at different temperatures ranging from 284 to $306 \mathrm{~K}$, showing apparent impact on the TS characteristics. Only the $\mathrm{VO}_{2}$ device was heated in the probe station during the temperature-elevated test, while the other components were all kept at room temperature. In order to further investigate the impact of temperature on the characteristics of $\mathrm{VO}_{2}$ memristor, critical paramters including $V_{\text {th }}$ and $V_{\text {hold }}$ are extracted. Figure 5c further shows $V_{\text {th }}$ and $V_{\text {hold }}$ values at different temperatures. One can see that both $V_{\text {th }}$ and $V_{\text {hold }}$ decrease as the temperature increases, which agrees with the switching behavior of $\mathrm{VO}_{2}$ observed in the previous study and variation in switching voltages originates from thermal processes induced by Joule heating and its dissipation. ${ }^{[43-46]}$ In order to exclude the possibility of stochastic fluctuations, we have systematically measured the cycle-to-cycle variation of the $\mathrm{VO}_{2}$ memristor under different temperatures (Figure S11). The experimental results in Figure S11 demonstrate that the temperature dependence of the threshold voltages 

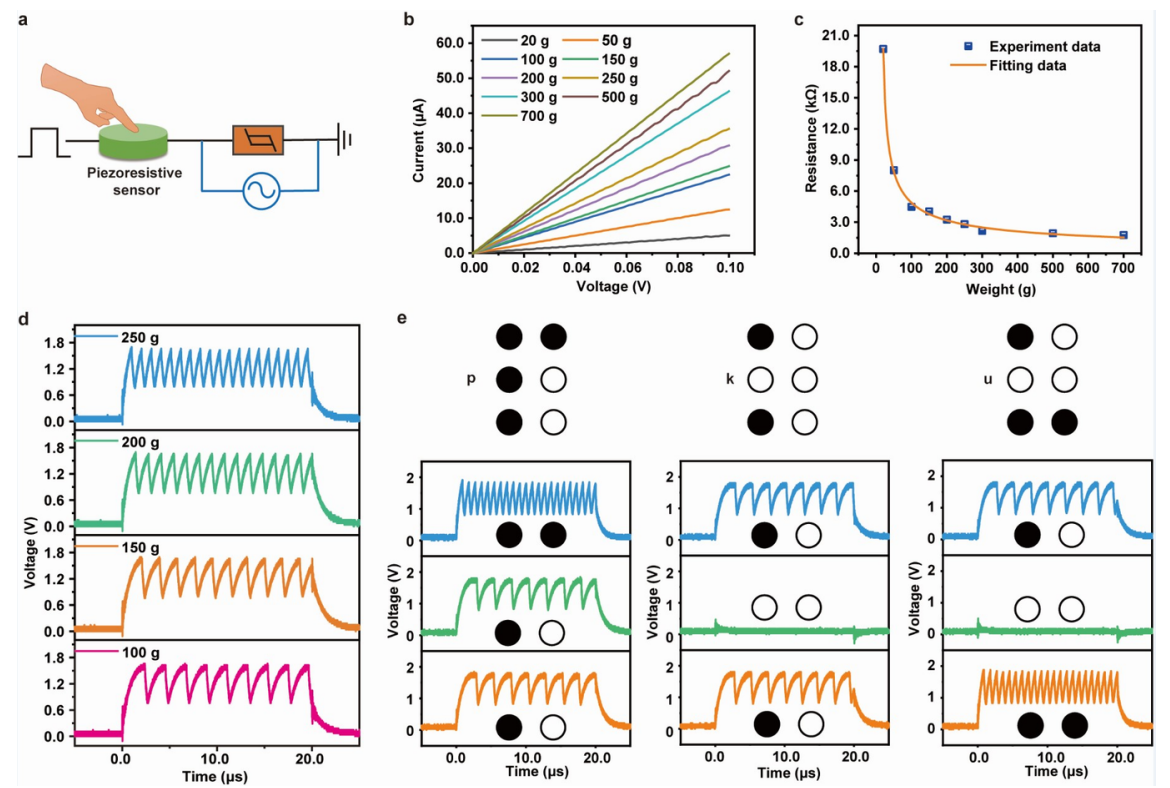

Figure 4: Characterization of the artificial haptic perception neurons. a) The circuit diagram of the haptic perception neuron. b) $I-V$ curves of the piezoresistive sensor with different applied pressures/weights. c) The output resistance of sensor as a function of weight. The solid line is fitting result by Equation (3). d) Neuronal response of the haptic perception neuron under a constant bias voltage $(5 \mathrm{~V}, 20 \mu \mathrm{s})$ and the influence of varying pressure. The output frequencies are $0.45,0.55,0.75$, and $0.95 \mathrm{MHz}$ at the pressures/weights of $100,150,200$, and $250 \mathrm{~g}$, respectively e) Recognition of braille characters ("p", "k", "u") using tactile sensing. Without convex patterns (that is, two sensors have no pressure), the output frequency is zero. With convex patterns both on the left and right (that is, two sensors both have pressure by applying $100 \mathrm{~g}$ ), the output frequency is $\sim 1.1 \mathrm{MHz}$. With convex patterns only on one of the left and right (that is, the only one of two sensors has pressure), the output frequency is $\sim 0.4 \mathrm{MHz}$.

in the $\mathrm{VO}_{2}$ memristor is much more significant than the parameter fluctuations and hence contribute to the temperature sensing capability of the sensory neuron. We have characterized the operation temperature range of our $\mathrm{VO}_{2}$ device. The results demonstrate that the TS characteristics remain stable at least below $0{ }^{\circ} \mathrm{C}$ but gradually disappear above $35{ }^{\circ} \mathrm{C}$ (Figure S12, Supporting Information), which could be related to the low phase transition temperature of $\mathrm{VO}_{2}\left({ }^{\sim} 340 \mathrm{~K}\right)^{[47]}$. This issue can potentially be addressed by using Mott system with higher phase transition temperature, such as $\mathrm{NbO}_{2}(\sim 1080 \mathrm{~K})^{[48]}$.

Lee et al. ${ }^{[44]}$ proposed a Joule heating model to point out that the relationship between $V_{\text {th }}, V_{\text {hold }}$ and temperature $(T)$. In this model, the mott transition occurs when the voltage-induced Joule heating is sufficient to raise the crystal temperature to the mott transition temperature. Assuming the heat obtained in the $\mathrm{VO}_{2}$ crystal is mainly due to the balance between resistive Joule heating and heat loss from the crystal to the environment via heat conduction, this relation can be expressed by the following simple heat equation:

$c \frac{d T}{d t}=-\frac{1}{R_{t h}}\left(T-T_{e}\right)+\frac{V_{V O_{2}} \cdot V_{V O_{2}}}{R}(4)$

where $C, T, R_{\mathrm{th}}$, and $R$ are the heat capacitance, temperature, effective thermal resistance, and resistance of the $\mathrm{VO}_{2}$ film. $V_{\mathrm{VO} 2}$ is the applied voltage to the $\mathrm{VO}_{2}$; film and $T_{\mathrm{e}}$ is the environmental temperature. Equation (4) is a first differential equation, where the relationship between $V_{\text {th }}, V_{\text {hold }}$ and $T_{\mathrm{e}}$ can be obtained by integrating (see Supporting Information), which shows the $T_{\mathrm{e}}$-dependence of $V_{\text {th }}$ and $V_{\text {hold }}$. Both $V_{\text {th }}$ and $V_{\text {hold }}$ decreased with increasing $T_{\mathrm{e}}$.

Furthermore, Figure 5d illustrates the oscillation frequency of the structure shown in Figure 5a as 
a function of $R_{\mathrm{L}}$ at different temperatures from 284 to $306 \mathrm{~K}$, when a constant voltage pulse $(5 \mathrm{~V}, 20 \mu \mathrm{s})$ is applied. The oscillation frequency generally increases linearly with decreased resistance, and the oscillation frequency is increased at evelated temperature when $R_{\mathrm{L}}$ is fixed (Figure $5 \mathrm{~d}$ ). Figure 5e further shows the oscillation waveforms at varied temperature from 284 to $306 \mathrm{~K}$, with constant $R_{\mathrm{L}}$ of $3.6 \mathrm{k} \Omega$ and the same input voltage pulse $(5 \mathrm{~V}, 20 \mu \mathrm{s})$. Once again the output oscillation frequency increases (from 0.3 to $0.8 \mathrm{MHz}$ ) as the temperature increases (from 284 to $306 \mathrm{~K}$ ). Since the oscillation is activated between $V_{\text {hold }}$ and $V_{\text {th }}$, the amplitude of oscillation decreases as the temperature increases, which is consistent with the results in Figure 5b,c. Therefore, the $\mathrm{VO}_{2}$ neuron in Figure 5a can realize temperature perception based on intrinsic thermal sensitivity of $\mathrm{VO}_{2}$, which is able to perceive temperature changes and serves as a temperature sensory neuron.
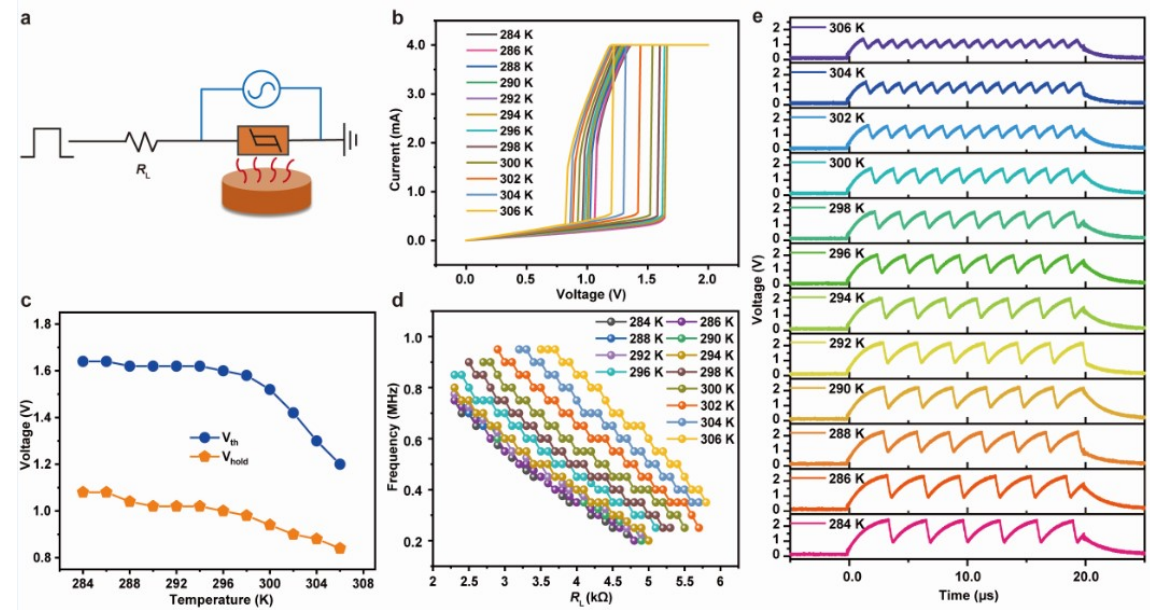

Figure 5: Characterization of the artificial temperature perception neurons. a) The circuit diagram of the temperature perception neuron. b) I-V curves of the $\mathrm{VO}_{2}$ volatile memristor with different temperatures. c) The temperature dependence of the threshold voltage (Vth) and hold voltage (Vhold) extracted from the I-V characteristic of Figure 5b. d) Oscillation frequency as a function of load resistance (RL) with different temperatures from 284 to $306 \mathrm{~K}$. e) Neuronal response of the temperature perception neuron under the varying temperature with $\mathrm{RL}$ of $3.6 \mathrm{k} \Omega$ and a constant bias voltage $(5 \mathrm{~V}, 20 \mu \mathrm{s})$. The output frequencies increases from 0.3 to $0.8 \mathrm{MHz}$ as the temperature increases from 284 to $306 \mathrm{~K}$.

\section{Haptic-temperature fusion and pattern recognition}

Multisensory neurons in the superior colliculus of the midbrain are revealed to directly integrate spikes from different senses to initiate a neuronal response to multimodal environmental events. ${ }^{[49-51]}$ For example, when two sensory stimuli appear at the same time, human generally shows higher sensitivity than the cases when the stimuli appear separately. This cognitive ability raises awareness and helps human make the right choices. Since the abovementioned sensory neuron is capable of detecting and encoding pressure and temperature inputs based on the voltage dividing effect and the intrinsic thermal sensitivity of $\mathrm{VO}_{2}$, respectively, it offers a potential platform for fusing and integrating tactile and temperature information directly in the same component, as shown in Figure 6a. The piezoresistive sensor provides the haptic perception while the temperature sensitivity of $\mathrm{VO}_{2}$ offers a mechanism for temperature perception, and both sensory inputs are converted into the oscillation frequency and amplitude of $\mathrm{VO}_{2}$ neuron, therefore achieving multisensory perception (the entire testing system is shown in Figure S13, Supporting Information). Indeed, experimental results in Figure 6b demonstrate that the $\mathrm{VO}_{2}$ sensory neuron can integrate haptic and temperature information. As examples, we use $\left(100 \mathrm{~g}, 27^{\circ} \mathrm{C}\right)$ to indicate an empty cup, $\left(200 \mathrm{~g}, 17^{\circ} \mathrm{C}\right)$ to indicate a cup with cold water, and $\left(200 \mathrm{~g}, 32^{\circ} \mathrm{C}\right)$ to indicate a cup with warm water. Figure $6 \mathrm{~b}$ shows that the abovementioned cases exhibit oscillation frequency of $0.5 \mathrm{MHz}, 0.6 \mathrm{MHz}$ and $1.1 \mathrm{MHz}$, respectively, showing 
the cross-modal perception capability of the $\mathrm{VO}_{2}$ neuron. The actual multisensory data collection processes are displayed in Supplementary Video 4-6. Although prior works have reported mapping of input signals into output frequencies, most of the works only realizes single mode perception. Here we take advantage of the intrinsic thermal sensitivity of metal-insulator transition in $\mathrm{VO}_{2}$ and its combination with a pressure sensor has led to multiple sensory perception.

In order to further illustrate the potential of the present $\mathrm{VO}_{2}$ neuron in multisensory fusion, we have tested a total of 11 combinations of different pressures and temperatures (Figure S14, Supporting Information), and the measurements were repeated 10 times for each individual combination to form a small dataset. A multilayer perceptron (MLP) network was constructed as shown in Figure 6c, which is composed of 20 hidden neurons and 11 output neurons, and the total 110 data samples were used to train the network to identify 11 different combinations using backpropagation. According to the experimental data in Figure S14, it can be found that only the oscillation frequency also cannot classify all 11 situations. Fortunately, it has been revealed that the temperature elevation also leads to variations in the amplitude of oscillation (Figure 5e). As a result, the maximum and mean of the output voltage signal have also been extracted (Figure S14) and included as input variables together with the oscillation frequency $(f)$. We have investigated three haptic-temperature input combinations, namely, $V_{\max }-f, V_{\text {mean }}-f$ and $V_{\max }-V_{\text {mean }}-f$, and the training is performed for 200 epochs. Figure 6d shows that the classification accuracies of the network are $86.03 \%$ and $83.25 \%$ based on $V_{\max }-f$ and $V_{\text {mean }}-f$ inputs after 200 epochs, respectively. When $V_{\text {max }}-V_{\text {mean }}-$ $f$ information is used as input, the classification accuracy can attain to $91.35 \%$ after 200 epochs, which has a better training performance. Figure 6e further shows a confusion matrix of the testing results for the 11 cases based on $V_{\max }-V_{\text {mean }}-f$ inputs. As a measure on the classification accuracy, the confusion matrix in Figure 6e displays the classification result in each column while the expected (actual) result in each row, where the number of instances is depicted by the color bar, demonstrating that the test inputs are well classified after training. 

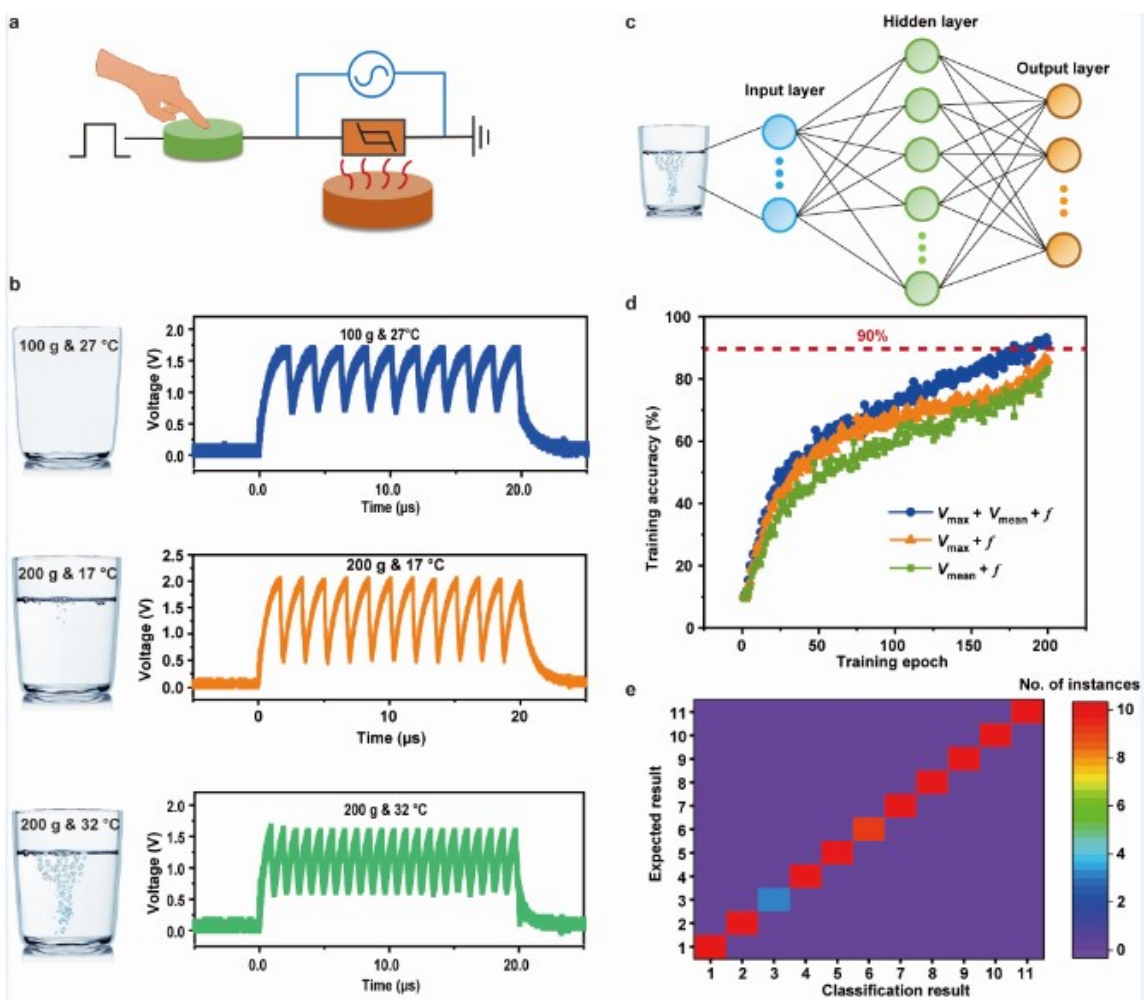

Figure 6: The haptic-temperature fusion based on $\mathrm{VO}_{2}$ volatile memristor and multilayer perceptron by simulation. a) The circuit diagram of the haptic-temperature fusion. b) Neuronal response of the haptic-temperature fusion under $100 \mathrm{~g} \mathrm{\&} 27^{\circ} \mathrm{C}, 200 \mathrm{~g} \& 17^{\circ} \mathrm{C}$ and $200 \mathrm{~g} \mathrm{\&} 32^{\circ} \mathrm{C}$. The output frequencies are $0.5,0.6$, and $1.1 \mathrm{MHz}$ at the haptic-temperature fusion under $100 \mathrm{~g} \& 27{ }^{\circ} \mathrm{C}, 200 \mathrm{~g} \& 17{ }^{\circ} \mathrm{C}$ and 200 g \& $32{ }^{\circ} \mathrm{C}$, respectively c) Schematic of the multilayer perceptron for pattern classification. d) Evolution of the training accuracy with training epoch. The line with blue circles is the training accuracy based on $V_{\text {max }}-V_{\text {mean }}-f$ inputs, which reaches $91.35 \%$ after 200 epochs. The line with orange triangles is the training accuracy based on $V_{\max }-f$ inputs, which reaches $86.03 \%$ after 200 epochs. The line with green squares is the training accuracy based on $V_{\text {mean }} f$ inputs, which reaches $83.25 \%$ after 200 epochs. e) Confusion matrix of the testing results, showing that the test inputs are well classified after training.

\section{Conclusion}

We have demonstrated an artificial multisensory integration neuron with haptic and temperature perception behaviors based on $\mathrm{VO}_{2}$ volatile memristor coupled with piezoresistive sensor. Such spiking neurons can be used to sense different pressure inputs and convert them into spike trains as a result of the voltage dividing effect between the piezoresistive sensor and $\mathrm{VO}_{2}$ memristor. Besides, the spiking neuron is also capable of sensing temperature, by taking advantage of the intrinsic thermal sensitivity of metal-insulator transition in $\mathrm{VO}_{2}$. Such spiking neuron is utilized to recognize Braille characters based on integration of multiple spatial correlated sensory stimuli, and the coordination of haptic and temperature sensory inputs give rise to recognition of multimodal haptic/temperature patterns. It should be noted that the $\mathrm{VO}_{2}$ based sensory neurons exhibit fast speed, acceptable cycle-to-cycle and device-to-device variations, but still show relatively high power consumption, owing to the relatively high $V_{\text {th }}(\sim 1.4 \mathrm{~V})$ and the low resistance of LRS 
$(\sim 500 \Omega)$. It is expected that the $V_{\text {th }}$ could be reduced by decreasing the length of the channel of the $\mathrm{VO}_{2}$ memristor (see detailed results in Figure S15, Supporting Information), and the on-state resistance of the device might be reduced by further optimizing the growth of the $\mathrm{VO}_{2}$ films. Our work offers new insights into neuromorphic perceptions and neuromorphic computing and the memristor based multisensory component shows great potential in cyborg systems, humanoid robotic systems, human-machine systems, and prosthetic system.

\section{Acknowledgements}

This work was supported by the National Key R\&D Program of China (2017YFA0207600), National Natural Science Foundation of China (61925401, 92064004, 61927901, 92164302), Project 2019BD002 and 2020BD010 supported by PKU-Baidu Fund, and the 111 Project (B18001). Y.Y. acknowledges the support from the Fok Ying-Tong Education Foundation and the Tencent Foundation through the XPLORER PRIZE.

\section{Conflict of interest}

The authors declare no conflict of interest.

\section{Supporting Information}

\section{References}

[1] F. McGlone, D. Reilly, Neurosci. Biobehav. Rev. 2010, 34, 148.

[2] V. E. Abraira, D. D. Ginty, Neuron 2013, 79, 618.

[3] E. A. Lumpkin, M. J. Caterina, Nature, 2007, 445, 858.

[4] C. Wan, P. Cai, M. Wang, Y. Qian, W. Huang, X. Chen, Adv. Mater. 2020, 32(15), 1902434.

[5] M. Wang, Y. Luo, T. Wang, C. Wan, L. Pan, S. Pan, K. He, A. Neo, X. Chen, Adv. Mater. 2021, 33, 2003014.

[6] T. Wang, M. Wang, L. Yang, Z. Li, X. J. Loh, X. Chen, Adv. Mater. 2020, 32, 1905522.

[7] Y. Khan, M. Garg, Q. Gui, M. Schadt, A. Gaikwad, D. Han, N. A. D. Yamamoto, P. Hart, R. Welte, W. Wilson, S. Czarnecki, M. Poliks, Z. Jin, K. Ghose, F. Egitto, J. Turner, A. C. Arias, Adv. Funct. Mater. 2016, 26, 8764 .

[8] W. Gao, S. Emaminejad, H. Y. Y. Nyein, S. Challa, K. Chen, A. Peck, H. M. Fahad, H. Ota, H. Shiraki, D. Kiriya, Nature 2016, 529, 509.

[9] Y. Ma, Y. Zhang, S. Cai, Z. Han, X. Liu, F. Wang, Y. Cao, Z. Wang, H. Li, Y. Chen, X. Feng, Adv. Mater. 2020, 32, 1902062.

[10] H. R. Lim, H. S. Kim, R. Qazi, Y. T. Kwon, J. W. Jeong, W. H. Yeo, Adv. Mater. 2020, 32, 1901924. 
[11] J. Zhu, T. Zhang, Y. Yang, R. Huang, Appl. Phys. Rev. 2020, 7, 011312.

[12] J. Li, Y. Yang, M. Yin, X. Sun, L. Li, R. Huang, Mater. Horiz. 2020, 7, 71.

[13] K. Yang, Q. Duan, Y. Wang, T. Zhang, Y. Yang, R. Huang, Sci. Adv. 2020, 6, eaba9901.

[14] Q. Duan, Z. Jing, X. Zou, Y. Wang, K. Yang, T. Zhang, S. Wu, R. Huang, Y. Yang, Nat. Commun. 2020, 11,339 .

[15] Z. Wang, S. Joshi, S. E. Savel'ev, H. Jiang, R. Midya, P. Lin, M. Hu, N. Ge, J. P. Strachan, Z. Li, Q. Wu, M. Barnell, G.-L. Li, H. L. Xin, R. S. Williams, Q. Xia, J. J. Yang, Nat. Mater. 2017, 16, 101.

[16] J.-T. Yang, C. Ge, J.-Y. Du, H.-Y. Huang, M. He, C. Wang, H.-B. Lu, G.-Z. Yang, K.-J. Jin, Adv. Mater. 2018, 30, 1801548.

[17] J. Zhu, Y. Yang, R. Jia, Z. Liang, W. Zhu, Z. U. Rehman, L. Bao, X. Zhang, Y. Cai, L. Song, Adv. Mater. 2018, 30, 1800195.

[18] X. Zhang, Y. Zhuo, Q. Luo, Z. Wu, R. Midya, Z. Wang, W. Song, R. Wang, N. K. Upadhyay, Y. Fang, F. Kiani, M. Rao, Y. Yang, Q. Xia, Q. Liu, M. Liu, J. J. Yang, Nat. Commun. 2020, 11, 51.

[19] L.Mennel, J. Symonowicz, S. Wachter, D. K. Polyushkin, A. J. Molina-Mendoza, T. Mueller, Nature 2020, 579,62 .

[20] F. Zhou, Z. Zhou, J. Chen, T. H. Choy, J. Wang, N. Zhang, Z. Lin, S. Yu, J. Kang, H.-S. P. Wong, Y. Chai, Nat. Nano. 2019, 14, 776.

[21] C. Wan, P. Cai, M. Wang, Y. Qian, W. Huang, X. Chen, Adv. Mater. 2020, 32, 1902434.

[22] B. Zhu, H. Wang, Y. Liu, D. Qi, Z. Liu, H. Wang, J. Yu, M. Sherburne, Z. Wang, X. Chen, Adv. Mater. 2016, 28, 1559 .

[23] J. H. Yoon, Z. Wang, K. M. Kim, H. Wu, V. Ravichandran, Q. Xia, C. S. Hwang, J. J. Yang, Nat. Commun. 2018, 9, 417.

[24] Y. Zang, H. Shen, D. Huang, C. A. Di, D. Zhu, Adv. Mater. 2017, 29, 1606088.

[25] C. Wan, G. Chen, Y. Fu, M. Wang, N. Matsuhisa, S. Pan, L. Pan, H. Yang, Q. Wan, L. Zhu, X. Chen, Adv. Mater. 2018, 30, 1801291.

[26] C. Wan, P. Cai, X. Guo, M. Wang, N. Matsuhisa, L. Yang, Z. Lv, Y. Luo, X. J. Loh, X. Chen, Nat. Commun. 2020, 11, 4602.

[27] C. Zhang, W. B. Ye, K. Zhou, H. Y. Chen, J. Q. Yang, G. Ding, X. Chen, Y. Zhou, F. Li, S. T. Han, Adv. Funct. Mater. 2019, 29, 1808783.

[28] Q. Wu, B. Dang, C. Lu, G. Xu, G. Yang, J. Wang, X. Chuai, N. Lu, D. Geng, H. Wang, L. Li, Nano Lett. 2020, 20, 8015 .

[29] F. Li, R. Wang, C. Song, M. Zhao, H. Ren, S. Wang, K. Liang, D. Li, X. Ma, B. Zhu, H. Wang, Y. Hao, ACS nano 2021, 15, 16422.

[30] X. Wu, E. Li, Y. Liu, W. Lin, R. Yu, G. Chen, Y. Hu, H. Chen, T. Guo, Nano Energy 2021, 85, 106000.

[31] H. Tan, Y. Zhou, Q. Tao, J. Rosen, S. van Dijken, Nat. commun. 2021, 12, 1120.

[32] T. Fu, X. Liu, H. Gao, J. E. Ward, X. Liu, B. Yin, Z. Wang, Y. Zhuo, D. J. F. Walker, J. J. Yang, J. Chen, D. R. Lovley, J. Yao, Nat. Commun. 2020, 11, 1861.

[33] M. A. Rahman, S. Walia, S. Naznee, M. Taha, S. Nirantar, F. Rahman, M. Bhaskaran, S. Sriram, Adv. Intell. Syst. 2020, 2, 202000094

[34] J. C. Stevens, J. E. Hooper, Percept. Psychophys. 1982, 32, 282. 
[35] Z. Shao, X. Cao, H. Luo, P. Jin, NPG Asia Mater. 2018, 10, 581.

[36] G. Stefanovich, A. Pergament, D. Stefanovich, J. Phys. Condens. Matter 2000, $12,8837$.

[37] M. M. Qazilbash, M. Brehm, B. G. Chae, P. C. Ho, G. O. Andreev, B. J. Kim, S. J. Yun, A. V. Balatsky, M. B. Maple, F. Keilmann, H. T. Kim, D. N. Basov, Science 2007, 318, 1750.

[38] C. K. Tee, A. Chortos, A. Berndt, A. K. Nguyen, A. Tom, A. Mcguire, Z. C. Lin, K. Tien, W. G. Bae, H. Wang, P. Wei, H. H. Chou, B. Cui, K. Deisseroth, T. N. Ng, Z. Bao, Science 2015, 350, 313.

[39] K. O. Johnson, Curr. Opin. Neurobiol. 2001, 11, 455.

[40] A. M. Fernandes, P. B. Albuquerque, Cogn. Process. 2012, 13, 285.

[41] O. Murtagh, B. Walls, I. V. Shvets, Appl. Phys. Let. 2020, 117, 063501.

[42] S. K. Nandi, S. K. Das, C. Estherby, A. Gentle, R. G. Elliman, J. Appl. Phys. 2020, $128,244103$.

[43] S. Rathi, J. H.Park, I. Y. Lee, J. M. Baik, K. S. Yi, G. H. Kim, J. Phys. D. 2014, 47, 295101.

[44] S. B. Lee, K. Kim, J. S. Oh, B. Kahng, J. S. Lee, Appl. Phys. Let. 2013, 102, 063501.

[45] O. Murtagh, B. Walls, I. V. Shvets, Appl. Phys. Let. 2020, 117, 063501.

[46] B. S. Mun, J. Yoon, S. K. Mo, K. Chen, N. Tamura, C. Dejoie, M. Kunz, Z. Liu, C. Park, K. Moon, H. Ju, Appl. Phys. Lett. 2013, 103, 061902.

[47] J. Del Valle, P. Salev, F. Tesler, N. M. Vargas, Y. Kalcheim, P. Wang, J. Trastoy, M. H. Lee, G. Kassabian, J. G. Ramírez, M. J. Rozenberg, I. K. Schuller, Nature 2019, 569, 388.

[48] J. Lee, J. Kim, T. Kim, H. Sohn, PHYS STATUS SOLIDI-R, 2021, 15, 2000610.

[49] M. A. Meredith, B. E. Stein, Science 1983, 221, 389.

[50] N. P. Holmes, C. Spence, Curr. Biol. 2005, 15, 762.

[51] B. A. Rowland, B. E. Stein, Front. Neurosci. 2008, 2, 33. 